\title{
Inserción de TIC en pedagogías del área de las humanidades en una universidad chilena
}

Insertion of ICT in pedagogies in the humanities area at a Chilean university Inserção de TIC em pedagogias na área de humanidades em uma universidade chilena

\author{
Juan Silva Quiroz \\ Universidad de Santiago de Chile, Chile \\ Autor referente: juan.silva@usach.cl
}

Historia Editorial

Recibido: 24/06/2017

Aceptado: 16/11/2017

\section{RESUMEN}

La inserción de las tecnologías de la información y comunicación (TIC) en la formación inicial docente (FID)es una demanda creciente que el sistema educativo y la sociedad realizan a las Instituciones de Educación Superior que forman docentes, pues ellas deberían generar las condiciones para que los futuros docentes hagan un uso efectivo de las TIC durante el ejercicio de la profesión docente. Con el propósito de conocer el nivel de uso de las TIC y competencias digitales de los y las estudiantes de las Facultades de Educación, con el fin de elaborar estrategias de apoyo para la integración efectiva de las TIC en la FID,Enlaces del Ministerio de Educación diseñó, validó y aplicó una

encuesta a estudiantes de tercer año de pedagogía de 12 universidades. Este trabajo presenta los resultados del estudio en los estudiantes de pedagogía de la Universidad de Santiago de Chile. Los resultados muestran que los estudiantes poseen acceso a diferentes tecnologías, sin embargo presentan un disímil uso. Presentan un adecuado uso técnico de las TIC y un deficiente uso en aspectos pedagógicos. Aprenden de TIC en forma personal o asignaturas afines, pero no aprenden como enseñar con TIC. Durante su proceso formativo en las asignaturas no reciben formación que les permita modelar usos pedagógicos de las TIC. 
Palabras clave: Formación inicial docente; Competencia digital docente; TIC; Pedagogía.

\begin{abstract}
The integration of information technology and communication (ICT) in initial teacher training is a growing demand that the education system and society made to educational institutions of higher education that form teachers, because it would create the conditions for future teachers make effective use of ICT for the exercise of the profession. In order to know the level of use of ICT and digital skills of the students of the Faculty of Education, to develop strategies to support the integration of ICT in your learning, links the Ministry of Education designed, validated and he applied a survey of
\end{abstract}

third-year students of 12 universities of pedagogy. This paper presents the results of the study on student teachers from the Faculty of Humanities of the University of Santiago, Chile.The results show that the students have access to different technologies however they have a dissimilar use. Theyhave adequate technical ICT use and poor educational aspects. ICT learning personally or related subjects, but do not learn how to teach with ICT. During the training process in the subjects not training to enable them to model pedagogical uses of ICT they are received.

Keywords: Initial teacher training; Teaching digital competence; ICT; Pedagogy

\section{RESUMO}

A inserção das tecnologias de informação e comunicação (TIC) na formação inicial de professores (FID) é uma demanda crescente que o sistema educacional e a sociedade fazem às instituições de ensino superior que treinam os professores, pois devem gerar as condições para que os futuros professores façam uso efetivo das TIC durante 0 exercício da profissão docente. Para conhecer o nível de uso das TIC e das competências digitais dos alunos das Faculdades de Educação, a fim de desenvolver estratégias de apoio para a efetiva integração das TIC no FID, Links do Ministério da Educação projetou, validou e aplicou uma pesquisa para estudantes de pedagogia de terceiro ano de 12 universidades. Este artigo apresenta os resultados do estudo na pedagogia estudantes da Universidade de Santiago do Chile. Os resultados mostram que os alunos têm acesso a diferentes tecnologias, no entanto, eles apresentam um uso diferente. Eles apresentam um uso técnico adequado das TIC e um uso reduzido em aspectos pedagógicos. Eles aprendem as TIC de forma pessoal ou assuntos relacionados, mas não aprendem a ensinar com as TIC. Durante o processo de treinamento nas matérias, não recebem treinamento que Ihes permita modelar os usos pedagógicos das TIC.

Palavras-chave: Formação inicial de profesores;Competência de ensino digital; TIC; Pedagogia 


\section{Las TIC y la Formación Inicial Docente}

os estudiantes se encuentran insertos en una sociedad altamente tecnologizada, interactiva y colaborativa, donde las aplicaciones informáticas les permite entretenerse, formarsey comunicarse. Esta exposición a las Tecnologías de la Información y Comunicación (TIC) también está cambiando las prácticas de acceso y producción de información, las que se realizan en ambientes hipertextuales modificando las maneras de comunicación e intercambio entre las personas (Area, Gros\& Marzal, 2008). Los jóvenes viven en una cultura de la interacción y su paradigma comunicacional se basa en la interactividad al usar un medio instantáneo y personalizable como Internet. Los estudiantes universitarios que acceden a un uso intensivo y creativo de las herramientas de Web Social, suelen adaptarse mejor a ambientes de enseñanza creativos en vez de tradicionales (Duart, Gil, Pujol \& Castaño, 2008). No hay una generación homogénea, se presentan diferentes perfiles derivados del acceso a los aparatos tecnológicos, las horas de exposición frente a ellos y los tipos de usos (Kennedy, Judd, Churchward, Gray, \& Krause, 2009). Se requiere, por tanto, docentes que hagan un uso innovador de las TIC para convertir al estudiante en un actor protagónico de su aprendizaje, permitiéndole opinar, interactuar y construir conocimiento en red.

El uso de las TIC en los procesos de formación, demandan en los estudiantes el manejo de las competencias digitales para el aprendizaje y en los futuros docentes las competencias digitales para aprender y enseñar con TIC. El futuro docente debe pasar por procesos donde él use las TIC para su propia formación y donde además adquiera las herramientas metodológicas para incorporarlas en su quehacer docente desde la didáctica y la pedagogía. Apoyando procesos de aprendizaje que favorezcan el constructivismo, aprendizaje significativo, autoaprendizaje, ubicuidad, aprendizaje colaborativo y autorregulación. 
La formación docente es un factor clave en la innovación educativa, máxime si esta se encuentra orientada al tratamiento y gestión adecuada de la información y a lo que se entiende como competencia digital, aspectos imprescindibles para la adecuada integración en la sociedad del conocimiento y el aprendizaje permanente. Existe consenso en la importancia y la premura en integrar las TIC en la Formación Inicial Docente (FID), pues aunque estos estudiantes sean usuarios habituales de las tecnologías, no implica que sean capaces de usarlas para apoyar su proceso formativo y enseñar utilizándolas. Se requiere formar docentes con los conocimientos y habilidades necesarios para la utilización de las TIC como un recurso en su proceso de enseñanza-aprendizaje, capaces de desarrollar las competencias digitales en sus estudiantes.

\section{Competencias y Estándares TIC para docentes}

El Parlamento Europeo define ocho competencias claves para el aprendizaje permanente, una de ellas es la Competencia Digital (CD). Entre las competencias que un ciudadano necesita para poder participar en la sociedad, la CD es considerada como destacada, transversal y de carácter instrumental ya que ayuda al desarrollo de otras competencias como la lingüística y la matemática (Ferrari, 2013).

La CD implica el uso crítico y seguro de las Tecnologías de la Sociedad de la Información para el trabajo, el ocio y la comunicación. Apoyándose en el uso de las TIC para recuperar, evaluar, almacenar, producir, presentar e intercambiar información, y para comunicar y participar en redes de colaboración a través de Internet (Unión Europea, 2006, pag.2).

Desde el punto de vista conceptual, la CD es entendida como la suma de las habilidades, conocimientos y actitudes en aspectos tecnológicos, informacionales, multimedia y comunicativos, dando lugar a una compleja alfabetización múltiple (Larraz, 2013). 
La CD es genérica, refiere a lo que cualquier ciudadano debe manejar respecto de las TIC. A partir de esta CD se derivan otras como la CD del estudiante, tanto de sistema escolar como universitario. A nivel de estudiante de Educación Superior, Larraz (2013)en su estudio sobre la competencia digital de los estudiantes universitarios, propone cuatro tipo de alfabetización: informacional; tecnológica; multimedial; comunicativa. Esteve, Duch yGisbert(2014) analizaron diferentes instrumentos para la evaluación de la competencia digital encontrando que la alfabetización tecnológica y la informacional son las dos más evaluadas, dejando a la alfabetización multimedia y comunicativa un papel menor. Señala Area (2010) que para que los estudiantes de educación superior puedan acceder a información valiosa y actualizada en su campo de estudio de investigación o de actividad profesional, así como para formarse bajo metodologías socioconstructivistas, el alumnado debe formarse en competencias informacionales y digitales.

La Competencia Digital Docente (CDD), considera que un docente competente digitalmente dispone de las habilidades, actitudes y conocimientos requeridos para promover un verdadero aprendizaje en un contexto enriquecido por las TIC. Para ello, deben ser capaces de utilizar la tecnología para mejorar y transformar las prácticas del aula y para enriquecer su propio desarrollo profesional e identidad (Fraser, Atkins\&Hall, 2013; INTEF, 2013). La CDD debe considerarse como un conjunto de destrezas y de conocimientos asociado al uso de las tecnologías que debe garantizar su buen uso en el ejercicio profesional docente.

Para orientar respecto a cuáles son los componentes de la CDD, así como para definir indicadores de cómo evaluarlas y estrategias para lograrlas, se han publicado en la última década diferentes iniciativas en diversos contextos entre ellos (Tabla 1) : International SocietyforTechnology in Education (ISTE, 2008), Estándares de competencia TIC para docentes (UNESCO,2008), DigiLitLeicesterLeicester City Council (Fraser et. al, 2013), Estándares TIC para FID (MINEDUC-ENLACES, 2008), 
Actualización de competencias y estándares TIC para la profesión docente (MINEDUC-ENLACES, 2011), Competencias TIC para el desarrollo profesional docente (Ministerio de Educación Nacional Colombia, 2013), Rúbrica de la competencia digital docente (Lázaro \&Gisbert, 2015),Marco común de competencia digital

docente

(INTEF,2013).

Tabla 1: Estándares/Indicadores TIC en FID

\begin{tabular}{|c|c|c|c|c|c|c|}
\hline $\begin{array}{l}\text { Marco } \\
\text { modelo }\end{array}$ & Institución & & Referencia & $\begin{array}{l}\text { Dimensiones } \\
\text { elementos CDD }\end{array}$ & I & Niveles / Enfoques \\
\hline NETS-S & ISTE & & ISTE (2008) & $\begin{array}{l}\text { Aprendizaje } \\
\text { creatividad } \\
\text { estudiantes } \\
\text { Experiencias } \\
\text { aprendizaje } \\
\text { evaluación } \\
\text { Trabajo } \\
\text { aprendizaje de } 1 \\
\text { era digital } \\
\text { Ciudadanía digita } \\
\text { y responsabilidad } \\
\text { Crecimiento } \\
\text { profesional } \\
\text { liderazgo }\end{array}$ & $\begin{array}{l}\text { y } \\
\text { de } \\
\text { de } \\
y \\
\text { y } \\
\text { la } \\
\text { tal } \\
\text { d } \\
\text { y }\end{array}$ & $\begin{array}{l}\text { Principiante } \\
\text { Medio } \\
\text { Experto } \\
\text { Transformador }\end{array}$ \\
\hline $\begin{array}{l}\text { Estándares de } \\
\text { competencia } \\
\text { TIC para } \\
\text { docentes }\end{array}$ & UNESCO & & $\begin{array}{l}\text { UNESCO } \\
(2008)\end{array}$ & $\begin{array}{l}\text { Política y visión } \\
\text { Plan de estudios } \\
\text { evaluación } \\
\text { Pedagogía } \\
\text { TIC } \\
\text { Organización } \\
\text { administración } \\
\text { Formación } \\
\text { profesional } \\
\text { docentes }\end{array}$ & $\begin{array}{l}y \\
y \\
\text { de }\end{array}$ & $\begin{array}{l}\text { Adquisición de } \\
\text { nociones básicas } \\
\text { Profundización del } \\
\text { conocimiento } \\
\text { Generación de } \\
\text { conocimiento }\end{array}$ \\
\hline DigiLitLeicester & $\begin{array}{l}\text { Leicester } \\
\text { Council }\end{array}$ & City & $\begin{array}{l}\text { Fraser et al. } \\
(2013)\end{array}$ & $\begin{array}{l}\text { Búsqueda, } \\
\text { evaluación } \\
\text { organización } \\
\text { Crear y compartir } \\
\text { Evaluación } \\
\text { feedback } \\
\text { Comunicación, } \\
\text { colaboración } \\
\text { participación } \\
\text { E-Seguridad }\end{array}$ & ir & $\begin{array}{l}\text { Principiante o de } \\
\text { entrada } \\
\text { Básico o nuclear } \\
\text { Desarrollador } \\
\text { Pionero } \\
\text { avanzado }\end{array}$ \\
\hline
\end{tabular}




\begin{tabular}{|c|c|c|c|c|c|}
\hline & & & \multicolumn{3}{|c|}{$\begin{array}{l}\text { identidad en línea } \\
\text { Desarrollo } \\
\text { profesional }\end{array}$} \\
\hline $\begin{array}{l}\text { Competencias } \\
\text { TIC para } \\
\text { docentes }\end{array}$ & $\begin{array}{l}\text { Min. Educación } \\
\text { Chile }\end{array}$ & $\begin{array}{l}\text { MINEDUC- } \\
\text { ENLACES } \\
(2008,2011)\end{array}$ & $\begin{array}{l}\text { Pedagógica } \\
\text { Técnica } \\
\text { Gestión } \\
\text { Social, ética } \\
\text { legal } \\
\text { Desarrollo } \\
\text { profesional }\end{array}$ & y & $\begin{array}{l}\text { Inicial } \\
\text { Elemental } \\
\text { Superior } \\
\text { Avanzado }\end{array}$ \\
\hline $\begin{array}{l}\text { Competencias } \\
\text { TIC para el } \\
\text { desarrollo } \\
\text { profesional } \\
\text { docente }\end{array}$ & $\begin{array}{l}\text { Ministerio de } \\
\text { Educación } \\
\text { NacionalColombia }\end{array}$ & $\begin{array}{l}\text { Ministerio de } \\
\text { Educación } \\
\text { Nacional(2013) }\end{array}$ & $\begin{array}{l}\text { Tecnológica } \\
\text { Comunicativa } \\
\text { Pedagógica } \\
\text { De Gestión } \\
\text { Investigativa }\end{array}$ & & $\begin{array}{l}\text { Explorador } \\
\text { Integrador } \\
\text { Innovador }\end{array}$ \\
\hline $\begin{array}{l}\text { Definición del a } \\
\text { Rúbrica de la } \\
\text { CDD }\end{array}$ & $\begin{array}{l}\text { ARGET } \\
\text { UniversitatRovira i } \\
\text { Virgili }\end{array}$ & $\begin{array}{l}\text { Lázaro, J.L. y } \\
\text { Gisbert, } \\
\text { M.(2015) }\end{array}$ & $\begin{array}{l}\text { Didáctica, } \\
\text { curricular } \\
\text { metodológica } \\
\text { Planificación, } \\
\text { organización } \\
\text { gestión } \\
\text { espacios } \\
\text { recursos } \\
\text { tecnológicos } \\
\text { digitales } \\
\text { Relacional, étic } \\
\text { seguridad } \\
\text { Personal } \\
\text { profesional }\end{array}$ & $\begin{array}{r}\text { y } \\
y \\
\text { de } \\
y \\
\\
\text { a y } \\
\text { y }\end{array}$ & $\begin{array}{l}\text { Principiante } \\
\text { Medio } \\
\text { Experto } \\
\text { Transformador }\end{array}$ \\
\hline $\begin{array}{l}\text { Marco común } \\
\text { de } \\
\text { competencia } \\
\text { digital docente }\end{array}$ & $\begin{array}{ll}\text { Ministerio } & \text { de } \\
\text { Educación } & \text { de } \\
\text { España } & \end{array}$ & $\begin{array}{l}\text { INTEF } \\
\text { Ministerio de } \\
\text { Educación de } \\
\text { España(2013) }\end{array}$ & $\begin{array}{l}\text { Información } \\
\text { Comunicación } \\
\text { Creación } \\
\text { contenidos } \\
\text { Seguridad } \\
\text { Resolución } \\
\text { problemas }\end{array}$ & $\begin{array}{l}\text { de } \\
\text { de }\end{array}$ & $\begin{array}{l}\text { Inicial } \\
\text { Medio } \\
\text { Avanzado }\end{array}$ \\
\hline
\end{tabular}

Fuente: Silva. et. al. 2016

En estos estándares/indicadores el foco de la CDD está puesto en aspectos: pedagógicos, desarrollo profesional, éticos y seguridad, búsqueda y manejo de información, así como en la creación y comunicación de contenidos. Presentan dos formas de abordar los estándares de las competencias TIC docente. Por una parte, 
están aquellos referentes centrados en los aspectos tecnológicos; mientras que en la otra, se encuentran los referentes centrados en los aspectos pedagógicos, para la integración de las TIC en la formación docente.

Los estándares están definidos para docentes en ejercicio, salvo el caso de Chile, que posee estándares TIC para la FID. Sin embargo, se observa que todas las propuestas presentan un nivel base (con diferentes denominaciones participante inicial, explorador, etc), siendo este nivel, el mínimo que debería presentar un estudiante egresado de formación inicial docente.

\section{Las TIC en la FID}

En la educación superior, las TIC se han ido incorporando desde una lógica más autónoma, con menores niveles de coordinación y dependencia respecto de los ministerios de Educación. En este contexto se encuentra la FID, que a pesar de constituir un nivel formativo de características heterogéneas, presenta una misión central en común: ser la incubadora de los futuros docentes que enseñarán en los respectivos sistemas educativos (Brun, 2011).

En un estudio sobre las TIC en la FID en América Latina, los principales resultados encontrados fueron: la política se ha centrado en entregar infraestructura y capacitación a los docentes de escuelas, se pensó que las instituciones sabrían integrar las TIC en la formación de los futuros docentes; la falta de sistematizar y compartir experiencias, que ayuden a insertar las TIC en el curriculum de FID; la necesidad de insertar las TIC en FID, en la región el 52\% cuenta con políticas formales, el $50 \%$ de los países cuenta en los ministerios o secretarías con áreas de Integración de TIC en FID (Brun, 2011). Chile es el único país en América Latina que cuenta con Estándares para la inserción de TIC en FID (MINEDUC-ENLACES, 2008). Sin embargo, a pesar de contar con estándares estos no han logrado permear los 
currículum, integrando en forma innovadora las TIC en las prácticas pedagógicas en la FID; en otros países de América Latina el tema es aún más incipiente.

Un estudio realizado en Latinoamérica muestra que las instituciones formadoras de docentes respecto a la inserción de TIC en FID, se encuentran en dos niveles: a)etapa inicial: enfocadas en definir una política institucional, capacitación docente y fortalecen la formación con TIC; b) etapa avanzada, cuentan con un proyecto para integrar las TIC con cierta trayectoria, sus esfuerzos se focalizan en integrar las TIC en forma transversal en el curriculum, establecer estándares para evaluar las competencias TIC al egreso para enseñar y aprender, e integrar la formación con la realidad escolar (Heep, 2012).En Chile se cuenta con distintas cantidades de asignaturas de TIC, en diferentes semestres de las carreras y centradas principalmente en alfabetización digital, se forma para usar TIC no para hacer con TIC, para pensar, aprender y enseñar con TIC (MINEDUC-ENLACES, 2008). Más allá de las asignaturas, se aboga por un uso transversal de las TIC en FID (Silva, 2012; Heep, 2012).

Las barreras para insertar las TIC en FID definen los aspectos que dificultan esta inserción. Donohoo (2004) señala como principales barreras: la facultad no modela el uso de tecnología; la dependencia en una sola asignatura de tecnología; el acceso a la tecnología; la falta de habilidad del estudiante de transferir sus habilidades tecnologías al aula. En este último aspecto se requiere que adquieran las competencias para usar las TIC para favorecer su propio aprendizaje y para integrarlas en las prácticas pedagógicas. Hepp(2012) señala que una de las barreras para integrar las TIC en la FID son las competencias digitales de los estudiantes. Una barrera importante y base es la actitud que el profesor adopta respecto a las TIC, la cual se relaciona con las creencias de los docentes y sus experiencias previas existentes(Aviram \& Tami, 2004). Otra barrera central es la formación de los formadores de FID en metodologías para insertar las TIC en la FID (Silva, 2012). 
A partir de la presunción de que existe un nuevo perfil de aprendices algunos organismos, como la OCDE, han impulsado un conjunto de estudios para intentar describir lo que efectivamente sucede con el uso de TIC en la FID, lo resultados indican que estas tecnologías no son usadas como un elemento importante en el desarrollo de los objetivos formativos de dichas carreras. Esto implica que, paradojalmente, a pesar de los requerimientos creciente del sistema educativo y/o de las orientaciones que la política pública impulsa para hacerse cargo pedagógicamente de estos medios, académicos y estudiantes de FID los utilizan marginalmente en sus desempeños (OCDE, 2009). Un estudio realizado en las universidades chilenas (Centro de Educación y Tecnología (CET) -Centro de Estudios y Politicas Pubicas en Educación (CEPPE), 2010), encontró que en la FID existe un proceso incipiente y poco definido de incorporación de las TIC, constatando que ésta aún transita por una etapa inicial de integración. Se puede inferir que, los estudiantes de formación docente vivencian pocas experiencias de tipo formal para aprovechar el potencial innovador de las tecnologías instaladas en los centros escolares. Los estudios sobre el aprendizaje informal de los estudiantes universitarios muestran (que los espacios formativos desconocen y desaprovechan el tipo y manera en que los estudiantes aprenden fuera de las aulas universitarias incluidos los de FID (Carrasco, Jadue, Letelier \& Oliva, 2012). Estudiar el uso que los estudiantes de FID realizan de las TIC a nivel personal y académico y a los que son expuestos en sus procesos de formación, son necesarios para desarrollar de mejor forma la CDD desde la FID.

\section{La investigación}

\subsection{Objetivos}

El objetivo de la encuesta fue conocer la percepción del nivel de uso de las Tecnologías de la Información y Comunicación (TIC) en la formación de los 
estudiantes de carreras de pedagogía de la Facultad de Humanidades (FAHU) de la Universidad de Santiago de Chile.

Las preguntas de investigación:

- Cuál es uso de las tecnologías a nivel personal y académico por parte de las estudiantes de las carreras de pedagogía de la FAHU.

- Cuál es la frecuencia en que los estudiantes de las carreras de pedagogía de la FAHU, utilizan algunas herramientas TIC, en situaciones de clase.

- Qué percepción tienen los estudiantes de las carreras de pedagogía de la FAHU respecto al uso pedagógico de las TIC por parte de sus formadores.

- Cómo evalúan los estudiantes de las carreras de pedagogía de la FAHU, sus habilidades para usar las TIC

- Qué percepción tienen los estudiantes de las carreras de pedagogía de la FAHU de la integración de las TIC en su futuro desempeño docente.

\subsection{Metodología}

\section{Los participantes}

A nivel nacional contestaron la encuesta 2.311 estudiantes de pregrado de las carreras de pedagogía impartidas por 12 Universidades participantes en la mesa de TIC en FID, instancia convocada y coordinada por ENLACES del Ministerio de Educación de Chile (MINEDUC). Una de ellas, la Universidad de Santiago de Chile (USACH).La muestra en la USACH, objeto de este estudió, contó con 110 estudiantes de tercer año de las carreras de pedagogía de la Facultad de Humanidades (Tabla 2), esto es Inglés, Historia, Castellano y Educación General Básica. 
Tabla 2: Representación de las carreras

Fuente: Elaboración propia

\begin{tabular}{lll}
\hline Carrera & Frecuencia & Porcentaje\% \\
\hline Castellano & 28 & $25,5 \%$ \\
General Básica & 17 & $15,5 \%$ \\
Historia & 33 & $30,0 \%$ \\
Inglés' & 32 & 29,1 \\
Total & 110 & 100 \\
\hline
\end{tabular}

Un $60,9 \%$ de los estudiantes, señalaron pertenecer al género femenino. El 97,25\% afirmó contar con acceso a Internet en su casa o lugar de residencia. Un 34,5 \% de los participantes señala tener 21 años.

Los estudiantes universitarios que formaron parte de esta investigación participaron de manera voluntaria y anónima luego de brindar su consentimiento. La administración del instrumento se realizó en un único encuentro presencial y de forma colectiva. Asimismo, se les informó que se trataba de un estudio del Ministerio de Educación para mejorar la formación en TIC en la FID, y que los datos relevados serían utilizados con fines exclusivamente académicos y éstos serían socializados en encuentros en la Universidad.

\section{El instrumento y análisis de datos}

El instrumento es una encuesta, diseñada y validada con expertos, en el marco de la mesa TIC en FID del MINEDUC. La encuesta se organizó en dos secciones a fin de solicitar datos a los estudiantes: Datos Generales y Aspectos Académicos. En la primera sección de la encuesta se solicitó a los estudiantes información demográfica: edad; semestre de carrera estaban cursando; género; acceso a internet en el lugar donde vivían; aparatos tecnológicos que utilizaban y su contexto de empleo. Además contempló el uso de dispositivos tecnológicos, las preguntas buscaron conocer qué dispositivos electrónicos poseían los estudiantes y si los empleaban en ambientes de 
aprendizaje, la escala va desde el no poseer la tecnología, usarla en la universidad y usarla en la sala de clases. En la sección antecedentes académicos, se solicitó a los estudiantes: señalar la frecuencia de uso de algunos recursos tecnológicos; evaluar la frecuencia en que sus docentes hacían uso de algunos recursos tecnológicos en la docencia. En ambos casos se evaluó con escala: nunca, rara vez, al menos una vez al mes, al menos una vez a la semana, en cada clase;para la autoevaluación de las habilidades para efectuar algunas tareas con TIC, se les presentó una lista de 16 tareas a realizar con TIC y cuatro alternativas de respuesta en una gradiente que señalaban diferentes grados de competencia. Las opciones de respuesta fueron: No se, Sé poco (necesito que me ayuden para hacer cosas simples), Sé suficiente (me manejo autónomamente en cosas simples, pero me interesan), Sé bastante (uso distintos servicios y aprendo solo cosas nuevas).Para la percepción sobre la integración de las TIC en su futura docencia, se consideró 10 afirmaciones respectivamente, evaluadas con escala liker de 5 niveles desde totalmente en desacuerdo a totalmente de acuerdo.

El análisis de datos se realizó usando software estadístico SPSS, obteniéndose las frecuencia y porcentajes para las preguntas de caracterización, y frecuencia, porcentajes, media y desviaciones típica para las preguntas de apreciación.

\section{Resultados}

\section{Uso de dispositivos tecnológicos}

Con la finalidad de conocer el uso de las TIC en la vida académica y personal de los estudiantes, se consultó por el acceso a determinada tecnologías y su uso en los estudios. Las opciones van, desde un mínimo de no poseer la tecnología a un máximo de usarla en el aula. La tecnología con mayor presencia es el computador portátil, un $72 \%$ de los estudiantes señalaron poseerlo, un 9\%hacer uso de esta tecnología en la universidad y un $9 \%$ en la sala de clases. Un 51\% indicaron poseer un Smartphone, un 
$20 \%$ señala usar esta tecnología en la sala de clases y un $3 \%$ en la universidad. La tecnología con menor presencia es el Tablet, un22\%afirmaron poseerla,3\% señalaronhacer uso de esta tecnología en la universidad y un $2 \%$ en la sala de clases.

\section{Utilización de TIC en clase por parte del estudiante}

Las tecnologías están presentes en la vida de los estudiantes, pero no necesariamente su uso se refleja en tareas educativas. Por esta razón se buscó determinar la frecuencia en que los estudiantes utilizan algunas herramientas TIC, en situaciones de clase (Tabla 3). Los resultados muestran que se usa más lo que se encuentra al alcance de la mano como el Smartphone y la tarea más común es buscar información.

Tabla 3: Utilización de TIC en clase

\begin{tabular}{|c|c|c|c|c|c|c|c|}
\hline & Nunca & RaraVez & $\begin{array}{l}\mathrm{Al} \\
\text { menos } \\
\text { una } \\
\text { vez al } \\
\text { mes }\end{array}$ & $\begin{array}{l}\text { Al } \\
\text { menos } \\
\text { una vez } \\
\text { a la } \\
\text { semana }\end{array}$ & $\begin{array}{l}\text { En } \\
\text { cadaclase }\end{array}$ & Media & Desv. \\
\hline $\begin{array}{l}\text { Software } \\
\text { presentaciones }\end{array}$ & $0,0 \%$ & $17,3 \%$ & $44,6 \%$ & $35,5 \%$ & $2,7 \%$ & 2,24 & 0,77 \\
\hline $\begin{array}{l}\text { Software } \\
\text { especifico }\end{array}$ & $49,1 \%$ & $34,3 \%$ & $6,5 \%$ & $9,3 \%$ & $0,9 \%$ & 0,79 & 0,99 \\
\hline Buscador & $2,8 \%$ & $11,0 \%$ & $8,3 \%$ & $42,2 \%$ & $35,8 \%$ & 2,97 & 1,07 \\
\hline Software & $12,7 \%$ & $52,7 \%$ & $17,3 \%$ & $12,7 \%$ & $4,6 \%$ & 1,44 & 1,02 \\
\hline Pizarra digital & $87,2 \%$ & $10,9 \%$ & $1,8 \%$ & $0,0 \%$ & $0,0 \%$ & 0,15 & 0,4 \\
\hline Cliquerastecleras & $82,1 \%$ & $9,4 \%$ & $3,8 \%$ & $2,8 \%$ & $1,9 \%$ & 0,33 & 0,84 \\
\hline Smartphones & $21,8 \%$ & $15,5 \%$ & $5,5 \%$ & $21,8 \%$ & $35,5 \%$ & 2,34 & 1,6 \\
\hline Otras Tic & $75,3 \%$ & $17,3 \%$ & $1,2 \%$ & $6,2 \%$ & $0,0 \%$ & 0,38 & 0,8 \\
\hline
\end{tabular}

Fuente: Elaboración propia

Un $42,2 \%$ estudiantes afirmaron que empleaban "al menos 1 vez por semana" buscadores de Internet y un $35,8 \%$ señala hacerlo "en cada clase". Respecto al Smartphone un $35,5 \%$, afirmaron emplearlo en cada clase, $21,8 \%$ señala usarlos "al menos 1 vez a la semana". Por el contrario los recursos menos usados fueron, la pizarra digital interactiva y tecleras. Un $1,8 \%$ señala usar las pizarras digitales 
interactivas una vez al mes y un $87,2 \%$ nunca. Las tecleras, el 1,9\% señala usarla en cada clase y un $82,1 \%$ señala no utilizar cliqueras o tecleras en clases.

\section{Utilización de algunos recursos tecnológicos por parte de los docentes}

El docente es un modelador en el uso de las tecnologías, de esta forma resulta interesante, pesquisar qué tecnologías usa el formador y con que frecuencia. En relación a la percepción de los estudiantes de la frecuencia de uso recursos tecnológicospor parte de sus profesores en el contexto de las clases (Tabla 4), los resultados reflejan un uso tradicional de las tecnologías, asociados a la clase magistral.

Tabla 4: Utilización de recursos tecnológicos por parte de los docentes

\begin{tabular}{|c|c|c|c|c|c|c|c|}
\hline & Nunca & RaraVez & $\begin{array}{l}\text { Al menos } \\
\text { una vez } \\
\text { al mes }\end{array}$ & $\begin{array}{l}\text { Al menos } \\
\text { una vez a } \\
\text { la } \\
\text { semana }\end{array}$ & $\begin{array}{l}\text { En } \\
\text { cada } \\
\text { clase }\end{array}$ & Media & Desv \\
\hline $\begin{array}{l}\text { Software } \\
\text { presentaciones }\end{array}$ & $0,0 \%$ & $1,8 \%$ & $5,5 \%$ & $44,6 \%$ & $48,2 \%$ & 3,39 & 0,68 \\
\hline $\begin{array}{l}\text { Software } \\
\text { especifico }\end{array}$ & $60,0 \%$ & $17,3 \%$ & $4,6 \%$ & $10,9 \%$ & $7,3 \%$ & 0,88 & 1,32 \\
\hline Software & $35,8 \%$ & $29,4 \%$ & $13,8 \%$ & $16,5 \%$ & $4,6 \%$ & 1,25 & 1,23 \\
\hline Pizarra digital & $88,9 \%$ & $6,5 \%$ & $1,9 \%$ & $1,9 \%$ & $0,9 \%$ & 0,19 & 0,65 \\
\hline $\begin{array}{l}\text { Cliqueras- } \\
\text { tecleras }\end{array}$ & $83,2 \%$ & $6,5 \%$ & $4,7 \%$ & $1,9 \%$ & $3,7 \%$ & 0,36 & 0,95 \\
\hline Otras Tic & $88,9 \%$ & $4,4 \%$ & $2,2 \%$ & $3,3 \%$ & $1,1 \%$ & 0,23 & 0,75 \\
\hline
\end{tabular}

Fuente: Elaboración propia

El $48,2 \%$ de los estudiantesafirmaron que sus docentes empleaban software de presentaciones multimedia "en cada clase", el $44,6 \%$ señalaron que sus docentes hacían uso de esta tecnología "al menos 1 vez por semana".En un segundo nivel de uso y bastante distante se encuentra software especifico de la materia y software de procesamiento de datos, con un $18,1 \%$ y $21,1 \%$ respectivamente, sumando las categorías en cada clase yun una vez por semana. En los recursos menos usados se 
encuentra la pizarra digital interactiva, un $2,8 \%$ señalan que sus profesores la usan todas las clases o al menos una vez por semana, un $88,9 \%$ reportan un uso nulo. Un $5,6 \%$ señala un uso frecuente de las cliqueras o tecleras, considerando su uso en cada clase o al menos una vez por semana, un $83,2 \%$ se decanta por un nulo uso.

\section{Autoevaluación de las habilidades para realizar tareas con TIC.}

Frente al uso de las TIC, es importante conocer cómo los estudiantes autoevalúen sus habilidades para efectuar tareas mediadas por TIC (Tabla 5). No necesariamente hay una relación directa con la realidad, pero al menos una percepción, que indica cuáles habilidades estarían mejor logradas y cuáles habría que reforzar.

Tabla 5: Autoevaluación de las habilidades para realizar tareas con TIC

\begin{tabular}{|c|c|c|c|c|c|c|}
\hline & No sé & $\begin{array}{l}\mathrm{Se} \\
\text { poco }\end{array}$ & $\begin{array}{l}\text { Se } \\
\text { suficiente }\end{array}$ & $\begin{array}{l}\text { Se } \\
\text { bastante }\end{array}$ & Media & Desv. \\
\hline Usar Proyector & $6,4 \%$ & $35,5 \%$ & $40,9 \%$ & $17,3 \%$ & 2,69 & 0,83 \\
\hline Buscar Info. en internet & $0,0 \%$ & $0,0 \%$ & $20,0 \%$ & $80,0 \%$ & 3,8 & 0,4 \\
\hline Procesador de texto & $0,0 \%$ & $0,9 \%$ & $33,6 \%$ & $65,5 \%$ & 3,65 & 0,5 \\
\hline Planilla de cálculo & $13,6 \%$ & $36,4 \%$ & $31,8 \%$ & $18,2 \%$ & 2,55 & 0,94 \\
\hline Presentador multimedia & $0,0 \%$ & $8,2 \%$ & $48,2 \%$ & $43,6 \%$ & 3,35 & 0,63 \\
\hline Aplicaciones web 2.0 & $18,2 \%$ & $29,1 \%$ & $34,6 \%$ & $18,2 \%$ & 2,53 & 0,99 \\
\hline Plataformas docentes & $26,4 \%$ & $38,2 \%$ & $28,2 \%$ & $7,3 \%$ & 2,16 & 0,9 \\
\hline $\begin{array}{l}\text { Software de } \\
\text { especialidad }\end{array}$ & $44,6 \%$ & $34,6 \%$ & $17,3 \%$ & $3,6 \%$ & 1,8 & 0,85 \\
\hline Redes sociales & $0,0 \%$ & $2,7 \%$ & $28,2 \%$ & $69,1 \%$ & 3,66 & 0,53 \\
\hline Pizarras digitales & $60,9 \%$ & $27,3 \%$ & $8,2 \%$ & $3,6 \%$ & 1,55 & 0,8 \\
\hline Tecleras o cliqueras & $74,1 \%$ & $12,0 \%$ & $9,3 \%$ & $4,6 \%$ & 1,44 & 0,85 \\
\hline Portafolios digitales & $47,3 \%$ & $20,0 \%$ & $22,7 \%$ & $10,0 \%$ & 1,95 & 1,05 \\
\hline Google docs & $3,6 \%$ & $17,3 \%$ & $32,7 \%$ & $46,4 \%$ & 3,22 & 0,86 \\
\hline $\begin{array}{l}\text { Búsqueda especializada } \\
\text { académica }\end{array}$ & $7,3 \%$ & $24,6 \%$ & $39,1 \%$ & $29,1 \%$ & 2,9 & 0,91 \\
\hline $\begin{array}{l}\text { Búsqueda en base de } \\
\text { datos bibliográfica }\end{array}$ & $10,9 \%$ & $30,0 \%$ & $41,8 \%$ & $17,3 \%$ & 2,65 & 0,89 \\
\hline $\begin{array}{l}\text { Búsqueda biblioteca } \\
\text { digital universitaria }\end{array}$ & $3,6 \%$ & $28,2 \%$ & $40,9 \%$ & $27,3 \%$ & 2,92 & 0,84 \\
\hline
\end{tabular}

Fuente: Elaboración propia 
Buscar información en Internet con un 100\% de valoración positiva - se bastante o se suficiente-, seguido de procesador con $99,1 \%$ y redes sociales con un $97,3 \%$, son las herramientas TIC que los estudiantes manifiestan tener más habilidades para su uso. Por el contrario pizarras digitales interactivas con un $88,2 \%$ de valoración negativa No se o se poco-, tecleras o cliqueras con $88,2 \%$ y software de la especialidad con $79,2 \%$, son las herramientas TIC donde los estudiantes manifiestan un bajo nivel de habilidades para su uso. Las aplicaciones pedagógicas específicas software de la especialidad y portafolio digital, reciben una alta valoración negativa con un $79,2 \%$ y $67,3 \%$ que señalan no saber o saber poco.

\section{Las TIC y su integración en la futura docencia.}

Conocer la percepción de los estudiantes respecto a la integración de las TIC en su futura docencia (Tabla 6), es un insumo importante para planificar la inserción de las TIC en la FID, al estar los alumnos en etapa final de la formación y en contacto con la realidad educativa, pueden percibir algunos retos que presentan la formación para integración de las TIC en docencia. Para efecto de análisis los resultados se han agrupado en los extremos positivos y negativos. 
Tabla 6: Integración de TIC en la futura docencia

\begin{tabular}{|c|c|c|c|c|c|}
\hline $\begin{array}{l}\text { En desacuerdo o muy en } \\
\text { desacuerdo }\end{array}$ & $\begin{array}{l}\text { En } \\
\text { desacuerdo } \\
o \text { muy en } \\
\text { desacuerdo }\end{array}$ & Indiferente & $\begin{array}{l}\text { De } \\
\text { acuerdo } \\
0 \quad \text { muy } \\
\text { de } \\
\text { acuerdo }\end{array}$ & Media & Devs. \\
\hline $\begin{array}{l}\text { El equipamiento TIC de la Facultad } \\
\text { para los estudiantes está en buen } \\
\text { estado }\end{array}$ & $49,1 \%$ & $22,7 \%$ & $28,2 \%$ & 2,66 & 1,05 \\
\hline $\begin{array}{l}\text { software para los estudiantes es } \\
\text { ficiente para las necesidades } \\
\text { adémicas. }\end{array}$ & $53,6 \%$ & $26,4 \%$ & $20,0 \%$ & 2,43 & 1,11 \\
\hline $\begin{array}{l}\text { Tengo suficientes competencias TIC } \\
\text { oara lo que requieren el auto- } \\
\text { aprendizaje }\end{array}$ & $23,6 \%$ & $16,4 \%$ & $60,0 \%$ & 3,42 & 1,13 \\
\hline $\begin{array}{l}\text { Debiera conocer mejor el uso de } \\
\text { software de productividad (tipo } \\
\text { procesador de texto, planilla } \\
\text { electrónica y presentador) }\end{array}$ & $11,8 \%$ & $14,6 \%$ & $73,6 \%$ & 3,95 & 1,13 \\
\hline $\begin{array}{l}\text { Considero que mis profesores hacen } \\
\text { sso adecuado de las TIC en clases }\end{array}$ & $27,3 \%$ & $30,0 \%$ & $42,7 \%$ & 3,18 & 1,09 \\
\hline $\begin{array}{l}\text { Considero que las TIC son } \\
\text { importantes para mi futuro laboral } \\
\text { como profesor/a }\end{array}$ & $5,5 \%$ & $8,2 \%$ & $86,4 \%$ & 4,19 & 0,93 \\
\hline $\begin{array}{l}\text { La Facultad debiera ofrecer una } \\
\text { asignatura especifica sobre usos } \\
\text { generales de TIC en educación }\end{array}$ & $2,7 \%$ & $4,6 \%$ & $92,7 \%$ & 4,53 & 0,79 \\
\hline $\begin{array}{l}\text { Las pizarras digitales se usan } \\
\text { regularmente en clases. }\end{array}$ & $94,6 \%$ & $2,7 \%$ & $2,7 \%$ & 1,33 & 0,71 \\
\hline $\begin{array}{l}\text { Las tecleras o cliqueras se usan } \\
\text { regularmente en clases } \\
\text { Considero que las TIC debieran }\end{array}$ & $90,9 \%$ & $7,3 \%$ & $1,8 \%$ & 1,32 & 0,71 \\
\hline $\begin{array}{l}\text { integrarse en todos o en la mayoría } \\
\text { de los cursos }\end{array}$ & $10,0 \%$ & $24,6 \%$ & $65,5 \%$ & 3,75 & 1,1 \\
\hline
\end{tabular}

El 92,7\% de los estudiantes piensa que la Facultad debería ofrecer una asignatura específica sobre usos generales de TIC en educación. El 86,4\% de los estudiantes considera que las TIC son importantes para su futuro laboral como profesor. A nivel de uso de herramientas el $73,6 \%$ cree que debiera conocer mejor el uso de software de productividad (tipo procesador de texto, planilla electrónica y presentador).Un 65,5\% considera que las TIC debieran integrarse en todos o en la mayoría de los cursos. Un $60 \%$ de los estudiantes considera tener las competencias necesarias en TIC para 
poder aprender sin ayuda. En las afirmaciones menos valoradas el $94,6 \%$ y $90,9 \%$ señalan respectivamente que las pizarras digitales y tecleras o cliqueras no son utilizadas en la sala de clase.

\section{Conclusiones}

Se observa que hay coincidencia entre los usos pedagógicos de las TIC que reportan los alumnos, con el que ellos perciben de sus docentes, coincidiendo en las TIC de mayor y menor uso. Esto nos muestra el efecto modelador en el uso de las tecnologías por parte de los docentes, los cuales deben contar con el acceso a estos recursos y las competencias para integrarlos en su docencia. Es necesario que las facultades de educación cuenten al menos con la tecnología que las políticas educativas en la materia hacen llegar a los centros educativos, en Chile, el Ministerio no dota de tecnología a las universidades que forman docentes.

Los resultados a nivel de autoevaluación nos muestran que los estudiantes se encuentran competentes en las competencias más básicas como búsqueda y procesador de textos, y más débiles en el uso de software disciplinarios y recursos tecnológicos como las pizarras digitales interactivas. Estos resultados coinciden con los encontrados por Gutiérrez y Serrano (2016) quienes desarrollarlo una investigación para evaluar la competencia digital de 134 estudiantes de pedagogía de la universidad de Murcia y encontraron que el alumnado participante se considera competente en los aspectos más básicos de la competencia digital. Los países en los cuales las políticas TIC en la educación, ha predominado un enfoque centrado en la inversión en infraestructura y en la capacitación en habilidades instrumentales, presentan resultados magros en materia de integración de las TIC en las prácticas pedagógicas. Los estudiantes manifiestan la necesidad de contar con una asignatura de TIC general y valoran el uso de las TIC en su futura labor profesional, así como esperarían un uso de ellas en todas o la mayoría de las asignaturas, de tal forma de egresar mejor 
preparados. Para satisfacer esta necesidad, se requiere en la formación de los futuros docentes, implementar en las mallas curriculares asignaturas especificas de TIC y favorecer su uso en forma transversal. En estos planes integrar las TIC generales, con otra especificas de las disciplinas e integrar recursos tecnológicos que podría encontrarse en la escuela, especialmente los que forman partes de políticas en informática educativa, como las pizarras digitales interactivas, las Tablet. Debería existir políticas TIC en FID, en las Instituciones de Educación Superior responsable de la formación docente, alineadas con las políticas ministeriales, que orienten la formación en TIC en la FID. Un aspecto clave para ir evaluando la eficacia de estos planes, es una evaluación sistemática del logro de las competencia digital docente en la FID, evaluaciones autenticas que vayan más allá de la autopercepción.

Se esperaría que los formadores de docentes modelen el uso de las TIC por parte de los futuros docentes. Sin embargo, de los resultados de este estudio, se puede desprender que los formadores, difícilmente propondrán estrategias de enseñanza que inserten en forma innovadora las TIC en sus prácticas docentes, las cuales suelen estar vinculadas a formas tradicionales de enseñanza y a un uso poco creativo de los recursos tecnológicos. Es necesario, formar a los formadores en metodología de enseñanza activas apoyadas por tecnología, implementando planes de evaluación acompañamiento.

Los resultados de este estudio muestran una realidad en una Universidad y en un área disciplinaría, las Humanidades, habría que investigar qué pasaría con estudiantes de otras áreas disciplinarías y en contextos universitarios diversos, con mayor o menor acceso a tecnología, como las universidades que acogen a estudiante de mejor situación económica y otras que reciben a los estudiantes de sectores más vulnerables especialmente en regiones. 


\section{Referencias}

Area, M. (2010). ¿Por qué formar en competencias informacionales y digitales en la educación superior?. En Competencias informacionales y digitales en educación superior [monográfico en línea]. Revista de Universidad y Sociedad del Conocimiento (RUSC). $\quad 7(2) . \quad$ Recuperado dehttp://www.redalyc.org/articulo.oa?id=78016225012

Area, M., Gros, B., \&Marzal, M. (2008).Alfabetizaciones y tecnologías de la información y la comunicación. Madrid: Síntesis.

Aviram, R., \& Tami, D. (2004). The Impact of ICT on education: the three opposed paradigms,the lacking discourse.Unpublished manuscript, Beer-Sheva University Israel. Recuperado dehttp://www.elearningeuropa.info/extras/pdf/ict_impact.pdf

Brun, M (2011). Las tecnologías de la información y las comunicaciones en la formación inicial docente de América Latina, Santiago: CEPAL.

Carrasco, R., Jadue, F., Letelier, M. \& Oliva, C. (2012). Estudio exploratorio sobre aprendizaje no formal e informal de estudiantes y egresados universitarios. Calidad de la Educación, 1er semestre (36), 150-183.

CET-CEPPE. (2010). Tecnologías de la información y de las comunicaciones en la formación inicial docente Reporte nacional del estudio TIC-FID 2009. Santiago: IIE-UFRO.

Donohoo, J (2004): A Review of the Literature Examining the Barriers to Technology Integration, Disponible en http://www.gecdsb.on.ca/d\&g/ICT/Literature\%20Review.htm

Duart, J., Gil. M., Pujol, M. \& Castaño, J. (2008), La Universidad en la sociedad RED, usos de la internet en educación superior, Barcelona: Ariel.

Esteve, F., Duch, J. \&Gisbert, M. (2014).Los aprendices digitales en la literatura científica: diseño y aplicación de una revisión sistemática entre 2001 y 2010. 
Pixel-Bit, Revista de Medios y Educación, 45, 9-21. http://dx.doi.org/10.12795/pixelbit.2014.i45.01

Ferrari, A. (2013). DIGCOMP:A frameworkfordeveloping and understanding digital competence in Europe. Retrievedfrom: http://ftp.jrc.es/EURdoc/JRC83167.pdf

Fraser, J., Atkins, L., \& Richard, H. (2013). DigiLit Leicester. Supportingteachers, promoting digital literacy, transforminglearning.Leicester City Council.

Gutiérrez, I. \& Serrano J. (2016). Evaluación y desarrollo de la competencia digital de futuros maestros en la Universidad de Murcia. New Approaches In EducationalResearch, 5(1), 53-59. DOI: 10.7821/naer.2016.1.152

Hepp, P (2012). Caracterización de buenaspracticas en formacióninicialdocente en TIC. Recuperado de http://ww2.educarchile.cl/UserFiles/P0001/File/Redesdocentes1.pdf

ISTE

(2008).

NETS-T

forTeachers:

NationalEducationalTechnologyStandardsforTeachers,

SecondEdition.

Washington, DC: ISTE (International SocietyforTechnology in Education)

INTEF (2013).Marco Común de Competencia Digital Docente, Ministerio de Educación de Educación Cultura y Deporte de España.Disponible en: http://goo.gl/8uWazW

Kennedy, G., Judd, T. S., Churchward, A., Gray, K., \& Krause, K.-L. (2009). Firstyearstudents' experienceswithtechnology: Are theyreally digital natives? AustralasianJournal of EducationalTechnology, 24(1), 108-122. DOI:10.14742/ajet.1233http://dx.doi.org/10.14742/ajet.1233

Larraz, V. (2013). La competència digital a la universitat. Tesi doctoral. Universitatd'Andorra. Identificador: TD-017-100006/201210.

Ministerio de Educación Nacional (2013). Competencias TIC para el Desarrollo Profesional Docente. Recuperado de http://goo.gl/WbqS9Lhttp://goo.gl/WbqS9L 
MINEDUC-ENLACES(2011). Actualización de Competencias y Estándares TIC en la Profesión Docente. Ministerio de Educación, Chile

MINEDUC-ENLACES(2008). Estándares TIC para la Formación Inicial Docente: Una propuesta en el contexto Chileno. Ministerio de Educación, Chile.

OCDE. (2009). ICT and initial teacher training.Paris: Centre for Educational Research $\begin{array}{lll}\text { and Innovation } & \text { Retrieved }\end{array}$ http://www.oecd.org/document/25/0,3343,en_2649_35845581_42236185_1_1_ 1_1,00.htmlhttp://www.oecd.org/document/25/0,3343,en_2649_35845581_4223 6185_1_1_1_1,00.html

Silva, J. (2012). Estándares TIC para la Formación Inicial Docente. Una política en el contexto chileno. EducationPolicyAnalysis Archives, 20 (7), 1-36. http://dx.doi.org/10.14507/epaa.v20n7.2012

Silva, J., Miranda, P., Gisbert, M., Morales, M. \& Onetto, A. (2016). Indicadores para evaluar la competencia digital docente en laformación inicial en el contexto Chileno - Uruguayo. Revista Latinoamericana de Tecnología Educativa (RELATEC), 15(3), 55-67. http://dx.doi.org/10.17398/1695-288X.15.3.55

UNESCO. (2008). Competency standards modules.ICT competency standards for teachers.Recuperado dehttp://unesdoc.unesco.org/images/0015/001562/156207e.pdf

Unión Europea (2006). Recomendación del Parlamento Europeo y del Consejo de 18 de diciembre de 2006 sobre las competencias clave para el aprendizaje permanente. En Diario Oficial de la Unión Europea. Recuperado de http://goo.gl/tXQDg8 
Formato de citación

Silva, J. (2017).Inserción de TIC en pedagogías del área de las humanidades en una Universidad Chilena. Psicología, Conocimiento y Sociedad, 7(2), 110-133. Disponible en:http://revista.psico.edu.uy/ 